\title{
Strategic Goals Analysis for Action Plan Against Unexpected Disaster in Shahid Beheshti Hospital, Shiraz, Iran
}

\section{SadrolahMahmoudi', Abdolkhalegh Keshavarzi ${ }^{2}$, Mohammad Kalantari Meibodi ${ }^{3 *}$}

'Emergency Medicine Specialist, BaqiyatallahUniversity of Medical Sciences, Tehran, Iran

${ }^{2}$ General Surgery Specialist, Burn and Wound Healing Research Center, Shiraz University of Medical Sciences, Shiraz, Iran

${ }^{3}$ Emergency Medicine Specialist, Trauma Research Center, ShahidRajaee (Emtiaz) Trauma Hospital, Shiraz University of Medical Sciences, Shiraz, Iran

Study Area: Shiraz, Iran

Coordinates: $29^{\circ} 37^{\prime} \mathrm{N} ; 52^{\circ} 32^{\prime} \mathrm{E}$

Key words: Disaster management,Hospital readiness, Emergency, Earthquake.

Introduction:

In order to manage treatment of injuries caused by natural and/or man-made disasters, it is necessary to organize and coordinate various organizations such as hospitals, fire station, police services, etc. (Drabek, 1985; Koenig et al., 1996). Hospitals should be prepared for all sorts of expected/unexpected crises (internal-external). Internal crises are an emergency that can lead to discontinuance of services, endanger the lives of personnel, patients and visitors, and sometimes require a rapid evacuation of the hospital (Koenig \& Schultz, 1994; Perry \& Lindell, 2003; A.C.E.P. , 2001). Nevertheless, the external crises occur due to the natural causes or human activities outside the hospital and many victims outside of the hospital need help. To deal with such emergency situation(s), there should exist various facilities, equipment, and personnel with mobility (Koenig et al., 1996; Waeckerle, 1991). As

\section{Abstract}

Hospitals as organizations with increasing demands in critical situations are always needed for strong management and skills. Therefore, it becomes necessary for every hospital to have a specific disaster management plan or emergency preparedness plan according to their resources, facilities, risks and special features to guarantee their self-sufficiency. This study aims to investigate the strategic goals for developing a plan of action against the crisis in one of our country's hospital based on a descriptive methodology. A customized observational checklist including 34 parts and 638 items was developed to assess different aspects of the Hospital readiness against disaster. The level of the Hospital readiness against disaster was evaluated using the checklist and then its content validity and reliability was validated.The assessment evidenced the lowest level of safety against the adapted partition status. The highest safety level was reported in favour of lighting system, installation unit, health, and safety education status, vaccination and infection control status, ICU-CCU sectors, obstetrics, and gynecology sectors and corpses maintenance status. Further, our findings showed that the degree of the hospital readiness is excellent. However, due to the requirement of more awareness towards the unexpected disasters, the more efforts on proper training and planning for the managers and authorities feel to be necessary in order to increase the readiness in a confrontation.

compared to the maximum chances of natural disasters in Iran (Iran ranks sixth among countries of the world) with respect to the appropriate therapeutic facilities available in the Iranian hospitals, lack of hospital-specif ic protocol against the crisis leads to increase of complicationsinduced probable events, the available funding and requirements in the hospital program to deal with the crisis of local hospitals is very less while compared with the hospitals of overseas. Hospital Disaster Planning (HDP) needs to be developed according to the existing conditions and the type of natural disaster the country Iran face. There are four steps involved in HDP which includes Prevention, Preparation, Reaction, and Return to Primary (Drabek, 1985; Irwin, 1989; Waeckerle, 1991). Development of HDP is not necessarily a costly plan. While justifying the imposition of high costs, financial loses, and physical injuries induced by the lack of hospital 
planning in dealing with natural or manmade disasters, the allocation of funds in this regard is completely reasonable and logical. Maneuvering is a common method for evaluating and monitoring the operating program for various crises and events in a community. Maneuvers can be run to evaluate different parts of a program such as telecommunications, command, rescue, etc.

Since the main objective of the disaster management program is to provide the most optimal and suitable services to the high number of injuries, the establishment of a specific plan must possess a scientific and precise management for all the stages (before, during and after measures in the form of predicting the type and timing of the disaster, necessary to form different groups and committees of accidents including medical and paramedical teams to take responsibility and monitor the precise implementation of relief activities, training the staff, and running different maneuvers to be prepared for dealing with disasters, evaluating and analyzing the improvements after these unforeseen events (Daneshmandi et al., 2014). The ultimate purpose of this study is to examine the various sources based on scientif ic hospitals as per the local needs in order to prevent the confusion of crisis managers, loss of resources and the decrease of injuries as well as proper hospital management in dealing with disasters.

\section{Methodology:}

This is a descriptive, cross-sectional study performed in Shahid Beheshti Hospital of Shiraz, Iran.The researcher has attempted to assess the degree of readiness of this Hospital. First of all a disaster, Operational Development notebook of Shahid Beheshti Hospital was prepared and the corresponding checklists were defined as primary standards of the hospital and the triage maneuver was run with a hypothetical patient scenario. The instrument tool used in this study was an observational checklist, whose reliability and validity was thoroughly reviewed. The checklist consisted of 638 items covering 34 domains with "Yes/No" options. It assessed the readiness of the facilities and human resources. The researchers completed the checklist as an observer. Scoring was done by assigning the score of 2 for Yes answer and 1 for No answer. Thereafter, the data were imported into IBM SPSS 21 and the descriptive tests (relative and absolute frequency) were used to analyze the results. The rate of positive responses to the survey questions reflects the hospital readiness. Table 1 shows how to score the readiness: we def ined five levels with 20 scales to determine each of the domains.

Table-1: The category of scoring of readiness of the Hospital

\begin{tabular}{lllll}
\hline Very weak & Weak & Medium & Good & Excellent \\
\hline o-20 & $\mathbf{2 1 - 4 0}$ & $41-60$ & $61-80$ & $81-100$
\end{tabular}
unexpected events). It must be through preventive shreds of evidence to develop an HDP in the domestic

Results:

As shown in Table-2, the readiness and safety of different parts were investigated in terms of the positive responses. The lowest amount of safety belonged to the partition status whereas lighting system, installation unit, health, and safety education status, vaccination and infection control status, ICU-CCU sectors, Obstetrics and Gynecology sector and corpses maintenance status are in satisfactory status (Fig.-1).

Table-2: The absolute and relative frequency of readiness and safety of the hospital

S.No. Readiness

YES NO TOTAL Readiness Abs.F* Rel.F** Abs.F* Rel.F** Abs.F* Rel.F** Level

1. The condition of emergency exit and stairs

$\begin{array}{lllllll}27 & 90 & 3 & 10 & 30 & 100 & \text { Excellent }\end{array}$

2. Safety against fire

$\begin{array}{lllllll}26 & 89.65 & 3 & 10.35 & 29 & 100 & \text { Excellent }\end{array}$

3. The condition of elevator
$29 \quad 93.55$
$\begin{array}{lll}6.45 \quad 31 & 100\end{array}$
Excellent

4. Gas supply system (medical and household)

$\begin{array}{crrrrrr}32 & 94.12 & 2 & 5.88 & 34 & 100 & \text { Excellent } \\ \text { Lighting system } & & & & & \end{array}$

5. Lighting system

$\begin{array}{lll}0 & 20 & 100 \quad \text { Excellent }\end{array}$

6. Electrical system status

$\begin{array}{lllllll}8 & 88.89 & 1 & 11.11 & 9 & 100 & \text { Excellent }\end{array}$

7. Installations unit condition

$\begin{array}{lllllll}6 & 100 & 0 & 0 & 6 & 100 & \text { Excellent }\end{array}$

8. Checking glasses and windows

$\begin{array}{lllllll}14 & 82.35 & 3 & 17.65 & 17 & 100 & \text { Excellent }\end{array}$

9. Duct and interior view of the building condition

$$
\begin{array}{lllllll}
8 & 72.73 & 3 & 27.27 & 11 & 100 & \text { Good }
\end{array}
$$

10. Exterior view of the building

$\begin{array}{lllllll}12 & 80 & 3 & 20 & 15 & 100 & \text { Good }\end{array}$

11. Parking condition

$\begin{array}{lllllll}7 & 70 & 3 & 30 & 10 & 100 & \text { Good }\end{array}$

12. Partitions condition

$$
\begin{array}{lllllll}
4 & 57.14 & 3 & 42.86 & 7 & 100 & \text { Middle }
\end{array}
$$

13. The condition of furniture and interior decoration

$$
\begin{array}{lllllll}
8 & 61.54 & 5 & 38.46 & 13 & 100 & \text { Good }
\end{array}
$$

14. Protection and safety of individuals and equipment

$$
\begin{array}{lllllll}
11 & 84.62 & 2 & 15.38 & 13 & 100 & \text { Excellent }
\end{array}
$$

15. Procurement condition (tent, food, medicine, etc.)

$$
\begin{array}{lllllll}
17 & 80.95 & 4 & 19.05 & 21 & 100 & \text { Good }
\end{array}
$$

16. Safe places for individuals, patients, equipment and ... $\begin{array}{lllllll}26 & 83.87 & 5 & 16.13 & 31 & 100 & \text { Excellent }\end{array}$

17. The condition of personal protective equipment

$$
\begin{array}{lllllll}
5 & 62.5 & 3 & 37.5 & 8 & 100 & \text { Good }
\end{array}
$$

\begin{tabular}{|c|c|c|c|c|c|c|}
\hline 25 & 86.21 & 4 & 13.79 & 29 & 100 & Excellent \\
\hline . Lanc & positio & & & & & \\
\hline 26 & 89.65 & 3 & 10.34 & 29 & 100 & Excellent \\
\hline The & ition & & $C \mathrm{C}$ & & & \\
\hline 21 & 87.5 & 3 & 12.5 & 24 & 100 & Excellent \\
\hline
\end{tabular}

18. The condition of health and safety education

$$
\begin{array}{lllllll}
10 & 100 & 0 & 0 & 10 & 100 & \text { Excellent }
\end{array}
$$

19. The condition of vaccination and infection control

$$
\begin{array}{lllllll}
12 & 100 & 0 & 0 & 12 & 100 & \text { Excellent }
\end{array}
$$

20. The level of safety in the operating room 
S.No. Readiness

$\begin{array}{cccc}\begin{array}{c}\text { YES } \\ \text { NOs.F* }\end{array} & \text { Rel.F** } & \text { TOTAL } & \text { Readiness } \\ \text { Abs.F* } & \text { Rel.F** } & \text { Abs.F* } & \text { Rel.F** Level }\end{array}$

23. The safety of CSR

$\begin{array}{lllllll}30 & 93.75 & 2 & 6.25 & 32 & 100 & \text { Excellent }\end{array}$

24. The condition of different sectors

$\begin{array}{lllllll}8 & 100 & 0 & 0 & 8 & 100 & \text { Excellent }\end{array}$

25. Especial investigation into ICU-CCU condition

$\begin{array}{lllllll}9 & 100 & 0 & 0 & 9 & 100 & \text { Excellent }\end{array}$

26. Especial investigation into orthopedic sector

$$
\begin{array}{lllllll}
4 & 80 & 1 & 20 & 5 & 100 & \text { Good }
\end{array}
$$

27. Especial investigation into obstetrics and gynecology sector

$\begin{array}{lllllll}8 & 100 & 0 & 0 & 8 & 100 & \text { Excellent }\end{array}$

28. The condition of safety of pharmacy \& medicine warehouse $\begin{array}{lllllll}34 & 94.44 & 2 & 5.56 & 36 & 100 & \text { Excellent }\end{array}$

29. The condition of radiology

$\begin{array}{lllllll}18 & 90 & 2 & 10 & 20 & 100 & \text { Excellent }\end{array}$

30. The condition of laboratory safety

$\begin{array}{lllllll}37 & 92.5 & 3 & 7.5 & 40 & 100 & \text { Excellent }\end{array}$

31. The condition of chicken safety

$\begin{array}{lllllll}31 & 91.18 & 3 & 8.82 & 34 & 100 & \text { Excellent }\end{array}$

32. The condition of the environmental health

$\begin{array}{lllllll}14 & 87.5 & 2 & 12.5 & 16 & 100 & \text { Excellent }\end{array}$

33. The condition of support units

$\begin{array}{lllllll}15 & 88.24 & 2 & 11.76 & 17 & 100 & \text { Excellent }\end{array}$

34. The condition of the corpses maintenance
4
100
o
$\begin{array}{lll}0 & 4 & 100\end{array}$
Excellent

Abs.F* - Absolute frequency; Rel.F**- Relative Frequency

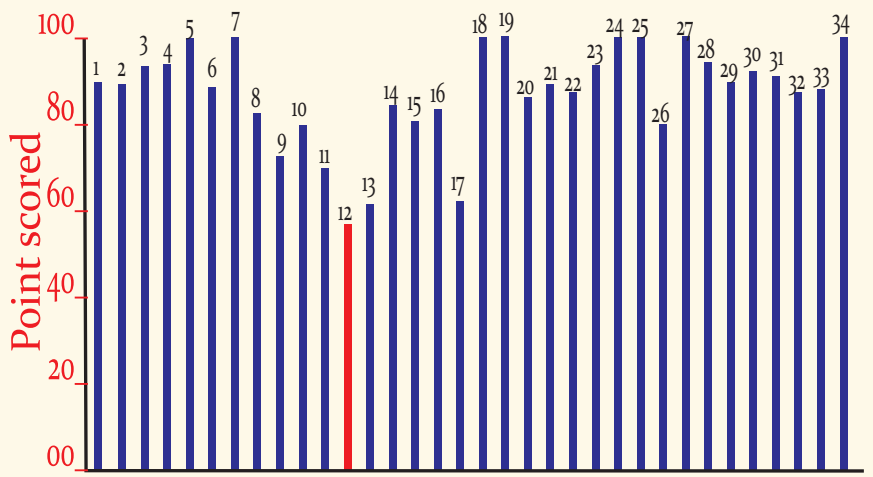

Figure-1: Comparison of the relative readiness and safety of different sectors of the hospital. As per Table-2, only the partitioning status was found at medium level. The average Hospital readiness was estimated $87.44 \%$ that was evaluated at the excellent level.

\section{Discussion:}

Proper security planning against accidents provides the safety of personnel and patients in critical situations and prevents undesired disturbances in health centers. Our results reveal that the hospital equipment is one of the most important issues in disaster preparedness and for the same, the staff should become familiar with relevant security issues and guidelines and must be updated continuously (Hsu et al., 2006; Koenig et al., 1996). Our data reflects that authorities have made considerable efforts in recent years to develop security guidelines, but there are still some deficiencies exist in the security equipment. However, there are differences between the security conditions in the confrontation of disasters and normal conditions but the better condition can be achieved by a special attention on this issue. The level of readiness in examining the status of training was $100 \%$ and was evaluated at its excellent level. The present result (the level of readiness) is higher than Amerion et al. (2010)'s study as $82.46 \%$, (good), Koari et al. (2005) as 77.8\%, (good); Hojat et al. (2008) as 66.69\% (good); Nasiripor et al. (2007) as 50.8\% (Medium); Hoseinishokoh et al. (2008) as $47.6 \%$ (Weak); Sedighi (2006) as $15-35 \%$, weak, and Ojaghi et al. (2009) as $17.9 \%$, very weak were more frequent.

In another study, Zaboli et al. (2006) demonstrated that $9.5 \%$ of hospitals have a regular education plan and $28.6 \%$ of hospitals enjoy appropriate educational facilities. Differences in the level of education indicate the viewpoints toward education in this field and the difference in manager's sensitivity to training the personnel. The surveys show that except for a few border towns, the majority of big cities have appropriate facilities and educational environment for implementation of theoretical classes, exercises, and practical maneuvers but there are no guidelines, instructions and detailed descriptions of the suitable training program to practice periodically. Soleimani (2006) pointed out that the hospital staff had been trained over different periods to deal with unexpected events and also educational pamphlets had been provided for colleagues and clients, but there was a lack of practical and operational awareness. The findings demonstrated that pieces of training related to staff readiness should be increased to decrease the stress in the confrontation with disasters which build up to $45 \%$ in personnel, especially in nurses (Amerion et al., 2010; Krajewski et al., 2005). Hence, we should pay special attention to the training the staff in order to improve their performance in the confrontation with disaster. The support unit obtained 88.24 score which was excellent and it was inconsistent with Amerion et al. (2010) as 81.21 (excellent) and was higher than the studies of Nasiripor et al. (2007) as 74.6\% (good), Hojat et al. (2008) as 68.97\% , (Good) and Hoseinishokoh et al. (2008) as $52.4 \%$ (medium).

In astudied hospital by Zaboli et al., (2006), the condition of facilities and necessities was medium while the level of equipment in the hospitals of Kermanshah evaluated by Ojaghi et al. (2009) was found to beweak.

Another important factor for managing the disaster is the parking condition, the readiness and the speed of operation in transportation and evacuation. In this regard, the readiness of the Shahid Beheshti hospital was found to be $70 \%$ which is a good level and it resembles the 
Amerion et al. (2010) study as $72 \%$ and higher than Hojat et al. (2008) as 39.63\%, Hoseinishokoh et al. (2008) and Koari et al. (2005) who found it as 33.3\% in their studies.

This level of readiness, particularly in big cities, does not enjoy a suitable degree which can be increased by a good plan or allocation of disaster condition-related facilities such as inputs equipped with time of disaster and the implementation of periodic maneuvers.It is very important to predict the problems and causes of the crisis in management because the management with accurate prediction can reduce the extent of the crisis (Nasiripor et al., 2007).

The results of the present study showed that the studied hospital was revealed at an excellent level in terms of structures and job description, but there are still some deficiencies in various sectors. To reach the maximum degree of readiness, one can make use of internal and international research results on improving the weaknesses in this field.

\section{Acknowledgements:}

The authors are thankful to the off icilas staff of Shahid Beheshti Hospital to cooperate with us during our study.

\section{References:}

A.C.E.P. (American College of Emergency Physicians.) (2001). American College of Emergency Physicians. ACEP emergency ultrasound guidelines-2001. Ann. Emerg. Med., $38(4): 470-481$.

Amerion, A., Delaavari, A. \& Teymourzadeh, E. (2010): Rate of preparedness in confronting crisis in three selected border hospitals. J. Mil. Med., 12(1):19-22.

Daneshmandi, M., Nezamzadeh, M. \& Zareiyan, A. (2014): Assessment the preparedness of selected hospital to deal with disasters in Tehran. Mil. Caring Sci., 1(1):28-35.

Drabek, T.E. (1985): Emergency management: the human factor. Mongraph, Vol. 2(3). Pub. by: Federal Emergency Management Agency. P. 46.

Hojat, M., Sirati, N.M., Khaghanizadeh, M. \& Karimi, Z.M. (2008): A survey of hospital disaster management in medical science universities. Daneshvar Med., 15(74), 1-10.

Hoseinishokoh, S., Arab, M., Rahimi, A., Rashidiyan, A. \& Sadremomtaz, N. (2008): Preparation rate the hospitals of Iran University of Medical Sciences and Health Services to the earthquake. L. Pub. Heal. faculty Res. Heal. Inst., 6: $(3,4): 61-77$. (Persian).
Hsu, E.B., Thomas, T.L., Bass, E.B., Whyne, D., Kelen, G.D. \& Green, G.B. (2006): Healthcare worker competencies for disaster training. BMCMed. Edu., 6(19).

Irwin, R. L. (1989): The incident command system (ICS). In der Heide, E.A. eds., Disaster Responses: Principles of Preparation and Coordination. Pub. by: Mosby, St Louis, Missouri, 133-163.

Koari, H., Moslehi, Sh. \& Panahi, A. (2005). Assessment of preparation rate hospitals of Shiraz medical university in disaster in 1384. Paper presented at the Second International Congress on Disaster Health and Crisis Management. First. Tehran.

Koenig, K.L., Dinerman, N. \& Kuehl, A.E. (1996): Disaster nomenclaturea functional impact approach: the PICE system. Acad. Emer. Med., 3(7):723-727.

Koenig, K.L. \& Schultz, C.H. (1994): Disaster medicine: advances in local catastrophic disaster response. Acad. Emer. Med. 1(2), 133-136.

Krajewski, M.J., Sztajnkrycer, M.D. \& Baez, A.A. (2005): Hospital disaster preparedness in the United States: new issues, new challenges. Internet J. Rescue Disaster Med., 4(2):

Nasiripor, A., Raeesi, P. \& Mahbobi, M. (2007): Readiness of hospitals in the border province of Kermanshah in dealing with cross-border crisis. J. Heal. Manag., 10(28):41-48.

Perry, R.W. \& Lindell, M.K. (2003): Preparedness for emergency response: guidelines for the emergency planning process. Disasters, 27(4):336-350.

Ojaghi, Sh., Norizad, S. \& Mahbobi, M. (2009): Assessment of preparation for Disaster in Center of educational and caring in Kermanshah University 1387. L. Kermanshah Med. Sci. Univer., 13 (3):267-274. (Persian)

Sedighi, aV. (2006): Assessment of preparation for Disaster. management of Center of Kashan about disaster 1385 . National Congress of strategies in Disaster management and Disaster. Unexpected promotion in Zanjan: 12. (Persian).

Soleimani, V. (2006): Assessment of preparation rate of Chamran hospital in Saveh in disaster in 1385 . Paper presented at the Second International Congress of crisis manegement in natural disaste. second. Tarvij.

Waeckerle, J.F. (1991): Disaster planning and response. N. Engl. J. Med.2 $324(12): 815-821$. 